\title{
Acoustic diffraction by deformed edges of finite length: Theory and experiment ${ }^{\text {a) }}$
}

\author{
Timothy K. Stanton and Dezhang Chu \\ Department of Applied Ocean Physics and Engineering, Woods Hole Oceanographic Institution, \\ Woods Hole, Massachusetts 02543-1053 \\ Guy V. Norton \\ Naval Research Laboratory, Stennis Space Center, Mississippi 39529I
}

(Received 30 June 2005; revised 16 August 2006; accepted 10 November 2006)

\begin{abstract}
The acoustic diffraction by deformed edges of finite length is described analytically and in the frequency domain through use of an approximate line-integral formulation. The formulation is based on the diffraction per unit length of an infinitely long straight edge, which inherently limits the accuracy of the approach. The line integral is written in terms of the diffraction by a generalized edge, in that the "edge" can be a single edge or multiple closely spaced edges. Predictions based on an exact solution to the impenetrable infinite knife edge are used to estimate diffraction by the edge of a thin disk and compared with calculations based on the T-matrix approach. Predictions are then made for the more complex geometry involving an impenetrable thick disk. These latter predictions are based on an approximate formula for double-edge diffraction [Chu et al., J. Acoust. Soc. Am. 122, 3177 (2007)] and are compared with laboratory data involving individual elastic (aluminum) disks spanning a range of diameters and submerged in water. The results of this study show this approximate line-integral approach to be versatile and applicable over a range of conditions.
\end{abstract}

(c) 2007 Acoustical Society of America. [DOI: 10.1121/1.2405126]

PACS number(s): 43.20.El, 43.30.Ft [AJMD] Pages: 3167-3176

\section{INTRODUCTION}

The subject of acoustic diffraction by finite deformed edges has been studied in previous investigations through analytical, numerical, and experimental methods. While all motivations have included advancing the fundamental understanding of the acoustic scattering processes, the applications were diverse and include understanding acoustic diffraction around noise barriers, seamounts, and seashells. Given the complexity and possible limitations of exact solutions [such as being constrained to certain canonical shapes (Bowman $e t$ al., 1987)], approaches are generally approximate and may be based, in part, on formally exact solutions to the wave equation for the infinitely long straight edge. For example, in the work of Medwin and colleagues, the Biot-Tolstoy solution was truncated in the time domain in order to estimate the acoustic diffraction by finite straight wedges (Medwin, 1981; Medwin et al., 1984). More recently, Svensson et al. (1999) incorporated the solution in a line integral to develop an approximate analytical time-domain formulation describing the diffraction by arbitrarily deformed edges of finite length. Also, Menounou and colleagues developed an approximate line-integral approach describing diffraction by edges and wedges of half-planes of arbitrary directivity functions (specific to the type of edge) and incident signal for both straight (Menounou et al., 2000) and jagged (Menounou and You, 2004) edges. One extension of the Medwin concept was the

\footnotetext{
Publication of this paper was delayed by request of author so that it would appear in the same issue with "Higher-order acoustic diffraction by edges of finite thickness" by Dezhang Chu, Timothy K. Stanton, and Allan D. Pierce.
}

construction of a deformed edge through an assemblage of wedges (Keiffer et al., 1994). Given the inherent inaccuracies of the approximate approaches, others have approached the problem through numerical methods (Kristensson and Waterman, 1982; Norton et al., 1993). In addition to the above cases involving impenetrable bodies, other studies have investigated the interaction of sound with elastic bodies that possess edges (Hefner, 2000: Hefner and Marston, 2001; 2002).

Although there has been a growing body of work involving diffraction by deformed finite-length edges, each work has been limited to either a particular geometry (i.e., shape of edge) or a particular infinite-edge solution as a basis, or both. Furthermore, there are relatively few comparisons of predictions of diffraction by deformed finite-length edges with numerical and experimental data. In this paper a general approximate analytical formula is derived to describe diffraction by deformed, finite-length edges (or multiple closely spaced edges). The formula is applied to the case of disks where the acoustic diffraction by the leading edge of various disks is studied theoretically, numerically, and experimentally. Disks of infinitesimal thickness (knife-edge) and finite thickness (double edge) are investigated for various diameters and orientations. As described below, the formulation is an advancement in terms of generality. Also, the numerical and experimental data are not only useful in comparisons with the theoretical predictions, but also contribute to the limited base of data associated with deformed finitelength edges. 


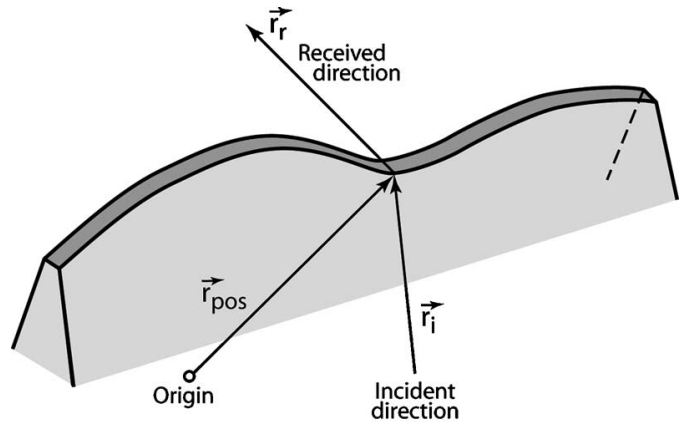

FIG. 1. Deformed truncated wedge of finite length.

The approximate analytical expression describing the diffraction by the deformed edge is derived using a formulation based on the case of infinitely long edges, but employing a line-integral along the length of the edge to account in an approximate manner for deformations of the edge (Fig. 1). Although this particular line-integral method is adapted from the deformed cylinder formulation in Stanton (1989), it is conceptually similar to other approaches such as Svensson et al. (1999) and Menounou et al. (2000). In fact, line-integral approaches have been used for more than one century and a brief review with comparisons between various approaches is presented in Menounou et al. (2000). Although similar conceptually, there are differences, however, involving generality and geometry. For example, the Svensson et al. formulas involve use of a particular infinite-edge solution and apply it to predict multiple-order diffraction between opposite edges of a disk. The Menounou et al. formulas involve an arbitrary infinite-edge solution and apply it to edges and wedges of half-planes. The approach described here is limited to diffraction from a single edge (or single group of closely spaced edges), but is written in a general form based on an arbitrary infinite-edge solution. Furthermore, it is written for an arbitrarily deformed edge of finite length. Note that although the Medwin et al. approach involved finite length edges, it incorporated one particular infinite edge formula (Biot-Tolstoy), while the approach in this paper is more general as it involves an arbitrary infinite edge formula. Since this new formulation is based on the diffraction by an infinitely long edge, it is generally applicable for conditions under which deformations in the edge are slowly varying and when the edge is near normal to the directions of the incident and diffracted signals (as in the Svensson et al. and $\mathrm{Me}-$ nounou et al. approaches).

As mentioned above, the general formula is evaluated for two cases of increasing complexity, each involving impenetrable disks. The first case involves the ideal geometry of the thin (knife-edge) disk which is based on an exact solution for the infinitely long knife edge presented in Morse and Ingard (1968). The predictions are then compared with calculations using the T-matrix approach (Kristensson and Waterman, 1982; Norton et al., 1993) for two diameters and a wide range of orientation angles. This is a relatively pure test of the deformed edge formulation as the only approximation in the comparison involves the fundamental approximation of the line integral being based, in part, on an infinite edge theory. The more complex case is then studied for disks of finite thickness. In this case, double-edge diffraction must be taken into account. The line-integral formula incorporates a double-edge diffraction formula from Chu et al. (2007), which is based on an extension of a formula in Pierce (1974) involving diffraction by an impenetrable infinitely long double edge. The results of these latter predictions are compared with laboratory measurements of broadband diffraction by the leading double edge of individual elastic disks (aluminum, submerged in water) of various diameters and over a wide range of orientation angles. In this latter case, the theory has three approximations. In addition to its inherent approximation of being based on an infinite edge theory, the infinite edge theory only approximately accounts for higherorder diffraction from the double edge. Also, the theory does not account for elastic effects.

This paper is organized as follows: In Sec. II, a general formula is derived describing the diffraction by finite-length deformed edges. The line-integral component of the formula is evaluated for the simple cases of a straight finite-length edge and a disk. In addition, the diffracted field by the leading edge is written explicitly for the case of a thin, impenetrable disk using the exact formula describing diffraction by an infinitely long knife-edge from Morse and Ingard (1968). In Sec. III, numerical calculations are described that are based on the T-matrix approach and are used to predict diffraction by a thin, impenetrable disk. In Sec. IV, a laboratory experiment is described in which the broadband diffraction by the leading double edge of a variety of individual thick, elastic disks submerged in water is measured. In Sec. V, results of the numerical calculations, laboratory experiment, and modeling using the line integral are presented and compared with each other. In Secs. VI and VII, the results are discussed and interpreted, and conclusions are made about the range of validity of the line-integral formulation.

\section{THEORY}

\section{A. General formulation}

The general approach toward describing the diffraction by deformed finite-length edges is based on the line-integral method used in Stanton (1989) to describe the scattering by deformed finite-length cylinders. In that case, the scattering per unit length of an infinitely long cylinder was incorporated into an integral along the length of the deformed cylinder. The integral took into account phase shifts due to deviations of the cylinder from a straight line. In a similar fashion, the diffraction per unit length from an edge is estimated and used to estimate the diffraction from deformed edges. This can apply to a single edge or assemblage of edges such as the double-edge case to be addressed later in this paper.

The line-integral approach begins by examining the radiated pressure from a line source. From Chap. 21 of Skudrzyk (1971), the differential pressure radiated from an infinitesimal section of an infinitely long line is

$$
d p_{l s}=\beta q \frac{e^{i k r_{r}}}{r_{r}} d z,
$$

where 


$$
\beta \equiv-\frac{i k \rho c}{4 \pi},
$$

$q$ is the volume flow per unit length of the line source, $k$ is the acoustic wave number $(=2 \pi / \lambda$, where $\lambda$ is the acoustic wavelength), $r_{r}$ is the distance between the receiver and an arbitrary point along the line, $d z$ is the differential length of the line which coincides with the $z$ axis, $\rho$ is the density of the surrounding fluid, and $c$ is the speed of sound of the fluid. The total pressure radiated by the line is calculated by integrating along its entire length,

$$
p_{l s}=\beta \int_{-\infty}^{\infty} q(z) \frac{e^{i k r_{r}}}{r_{r}} d z
$$

where $q$ is assumed to vary as a function of $z$.

At this point, the radiation from a line source has been formulated in terms of the volume flow per unit length. In this deformed-edge approximation, the radiation will now be treated as the reradiation due to the diffraction by a single, infinitely long straight edge or assemblage of closely spaced, infinitely long straight edges. The diffraction is bistatic and the source and receiver are in the same plane whose normal is the edge, which is still straight at this point in the derivation.

The deformed-edge formulation depends on whether the infinite edge formulation is based on use of a plane-wave source or a point source. A detailed derivation will first be given for the point-source-based infinite edge formulation. At the end of the derivation, details of the plane-wave-based formulation will be briefly summarized. For a point source a distance $r_{i}$ away from the edge at position $z$, the volume flow can be written as

$$
q(z)=f^{(\mathrm{PS})}(z) \frac{e^{i k r_{i}}}{r_{i}}
$$

where now $f^{(\mathrm{PS})}(z)$ is the component of $q$ due to the (point source) diffraction by the edge and the term $r_{i}^{-1} \exp \left(i k r_{i}\right)$ accounts for the spreading of the incident wave from the source and its phase shift. Integrating Eq. (3) and assuming that $f^{(\mathrm{PS})}(z)$ varies slowly with $z$ compared with $k r_{i}$ and $k r_{r}$ allows the stationary phase approximation to be used. Under this condition, the integral is approximately

$$
\begin{aligned}
P_{\text {diff }}^{(\infty, \mathrm{PS})} \simeq & \beta f^{(\mathrm{PS})}(0)(2 \pi)^{1 / 2}\left[k r_{i}^{(0)} r_{r}^{(0)}\left(r_{i}^{(0)}+r_{r}^{(0)}\right)\right]^{-1 / 2} \\
& \times e^{i k\left(r_{i}^{(0)}+r_{r}^{(0)}\right)} e^{i \pi / 4},
\end{aligned}
$$

where $r_{i}^{(0)}$ and $r_{r}^{(0)}$ are the shortest distances between the source and receiver, respectively, and the edge. As mentioned above, the expression for radiation, $p_{l s}$, in Eq. (3) has been replaced by the reradiation or diffraction $P_{\text {diff }}^{(\infty, P S)}$ by an infinitely long edge and due to a point source.

From this equation, $f^{(\mathrm{PS})}(0)$ is

$$
\begin{aligned}
f^{(\mathrm{PS})}(0)= & \beta^{-1}(2 \pi)^{-1 / 2}\left[k r_{i}^{(0)} r_{r}^{(0)}\left(r_{i}^{(0)}+r_{r}^{(0)}\right)\right]^{1 / 2} \\
& \times e^{-i k\left(r_{i}^{(0)}+r_{r}^{(0)}\right)} e^{-i \pi / 4} P_{\mathrm{diff}}^{(\infty, \mathrm{PS})} .
\end{aligned}
$$

In order to estimate the diffracted field from a finite-length deformed edge, or assemblage of closely spaced edges, this expression for $f^{(\mathrm{PS})}(0)$ is used to estimate the volume flow in an expression similar to Eq. (3). However, $q$ is only known (and approximately, at that) for $z=0$. Assuming that the component of the edge that dominates the diffraction is the one involving normal incidence (e.g., $z=0$ for the straight edge), the integral is approximated by replacing $f^{(\mathrm{PS})}(z)$ with $f^{(\mathrm{PS})}(0)$. This approximation restricts the geometries to those deformed edges with at least one section that involves normal incidence (such as at the midpoints of the leading and trailing edges of a disk). The phase $\operatorname{shift} \exp \left(i k r_{i}\right)$ in $q$ is still allowed to vary within the integral as the phase shifts play a significant role in the diffracted signal. Replacing $q(z)$ in Eq. (3) with $f^{(\mathrm{PS})}(0) r_{i}^{-1} \exp \left(i k r_{i}\right)$, and evaluating $\exp \left(i k r_{i}\right)$ and $\exp \left(i k r_{r}\right)$ in the far field, the diffracted field due to a finitelength deformed edge is, approximately,

$$
\begin{aligned}
P_{\mathrm{diff}} \simeq & P_{\mathrm{diff}}^{(\infty, \mathrm{PS})}(2 \pi)^{-1 / 2}\left[k\left(r_{i}^{(T)}+r_{r}^{(T)}\right) /\left(r_{i}^{(T)} r_{r}^{(T)}\right)\right]^{1 / 2} e^{-i \pi / 4} \\
& \times \int_{\mathbf{r}_{\mathrm{pos}}} e^{i k r_{\mathrm{pos}}\left(\hat{r}_{i}-\hat{r}_{r}\right) \cdot \hat{\mathrm{r}}_{\mathrm{pos}} \mid} d \mathbf{r}_{\mathrm{pos}} \mid
\end{aligned}
$$

where $\hat{r}_{i}, \hat{r}_{r}$, and $\hat{r}_{\text {pos }}$ are unit vectors (indicated by the " "» symbol) for the incident field, diffracted field toward the direction of the receiver, and position of the edges, respectively. For this finite-length geometry, the outer dimensions are small compared with $r_{i}$ and $r_{r}$ so that the amplitude dependence upon $r_{i}$ and $r_{r}$ could be taken outside the integral and be replaced by the terms $r_{i}^{(T)}$ and $r_{r}^{(T)}$, which correspond to the distance from the source and receiver, respectively, to a fixed point on or near the target (i.e., the origin). The term $P_{\text {diff }}^{(\infty, \mathrm{PS})}$ is evaluated using these latter distances. Furthermore, in this far-field approximation, the outer dimensions of the object are smaller than the first Fresnel zone of the transceiver. The term $\left|d \mathbf{r}_{\text {pos }}\right|$ replaces $d z$ to allow for an arbitrary deformation of the edge.

Equation (7) is a general approximate expression predicting the far-field diffraction by a deformed finite-length edge or edges. The formula is general both with respect to the facts that the edge is of arbitrary deformation and that the approach is not specific to any particular infinite-edge formulation (i.e., $\left.P_{\text {diff }}^{(\infty)}\right)$, with the exception that the infinite-edge formulation involves use of a point source. For the case in which the infinite-edge formulation involves a plane-wave source, there will be no phase shift $\left(\exp \left[i k r_{i}\right]\right)$ or spreading $\left(r_{i}^{-1}\right)$ terms in Eq. (4), simplifying that equation to $q(z)$ $=f^{(\mathrm{PW})}(z)$. Here, the phase of the incident plane wave is assumed to be uniformly zero along the straight axis. The only spreading term will involve $r_{r}$ for the point receiver. Following the same steps in the derivation as for the point-sourcebased formulation, the far-field diffraction by a deformed finite-length edge or edges is

$$
\begin{aligned}
P_{\mathrm{diff}} \simeq & P_{\mathrm{diff}}^{(\infty, \mathrm{PW})}\left(k / 2 \pi r_{r}^{(T)}\right)^{1 / 2} e^{-i \pi / 4} \int_{\mathbf{r}_{\mathrm{pos}}} e^{i k r_{\mathrm{pos}}\left(\hat{r}_{i}-\hat{r}_{r}\right) \cdot \hat{r}_{\mathrm{pos}}} \\
& \times\left|d \mathbf{r}_{\mathrm{pos}}\right| .
\end{aligned}
$$

This plane-wave-based formulation is quite similar to the corresponding point-source-based formulation given in Eq. (7), with the major difference being the dependence of $P_{\text {diff }}$ in Eq. (7) on the distance $r_{i}^{(T)}$ associated with the point source. 
The most significant approximation associated with both Eqs. (7) and (8) concerns the fact that the derivations are based on the diffraction from an infinitely long straight edge or edges. As with the deformed cylinder approach, these formulations will only be valid for smooth deformations in the edge and for angles of incidence near normal to the edge. However, for certain objects, this latter restriction is relaxed. As will be seen in later sections, for cases such as the disk, although there are angles of incidence along the perimeter well away from normal, errors associated with those angles do not contribute significantly to the estimated diffracted field since the contributions from the first Fresnel zone, which involve normal and near-normal incidence, dominate the diffraction.

\section{B. Special cases}

The general formulation in Eq. (8) is evaluated for two simple geometries, the straight finite-length edge(s) and disk.

\section{Straight finite-length edge(s)}

In the case of the straight edge(s) of length $L$, the integral in Eq. (8) is evaluated over the range $-L / 2$ to $+L / 2$ with the origin at the center of one of the edges. In this case, the approach is valid for scattering geometries where effects due to the ends of the target are not appreciable. Since a finite edge in this formulation is the finite-length portion of a semiinfinite strip, geometries where appreciable diffraction occurs by the edge of the semi-infinite portion of the strip must be avoided for this formulation to apply. The integral in Eq. (8) is equal to the product of the length of the edge(s) and a sinc function which describes the orientation dependence of the diffraction,

$$
P_{\text {diff }}=P_{\text {diff }}^{(\infty, \mathrm{PW})}\left(k / 2 \pi r_{r}^{(T)}\right)^{1 / 2} e^{-i \pi / 4} L \frac{\sin \Delta}{\Delta},
$$

where $\Delta=\frac{1}{2} k L\left(\hat{r}_{i}-\hat{r}_{r}\right) \cdot \hat{r}_{\text {edge }}$ for the bistatic case and $\Delta$ $=k L \cos \gamma$ for the case of backscatter $\left[\hat{r}_{\text {edge }}\right.$ is the unit direction vector of the edge and $\gamma$ is the angle between the direction of the incident field and the axis of the edge(s)]. This result shows that the diffraction by a finite-length edge(s) is proportional to the length of the edge(s). Furthermore, the orientation dependence of the diffraction is approximated by the sinc function.

\section{Disk}

In the case of the disk, first the general solution is expressed for the case of backscatter for this geometry, then an explicit expression is written for the case of a thin impenetrable disk. Finally, high-frequency approximations are made for the leading and trailing edges of a disk.

a. General expression for backscatter From the geometry defined in Fig. 2, the vectors in Eq. (8) are given as

$$
\begin{aligned}
& \mathbf{r}_{\text {pos }}=a \cos \phi \hat{i}+a \sin \phi \hat{j}, \\
& d \mathbf{r}_{\text {pos }}=(-a \sin \phi \hat{i}+a \cos \phi \hat{j}) d \phi, \\
& \left|d \mathbf{r}_{\text {pos }}\right|=a d \phi,
\end{aligned}
$$

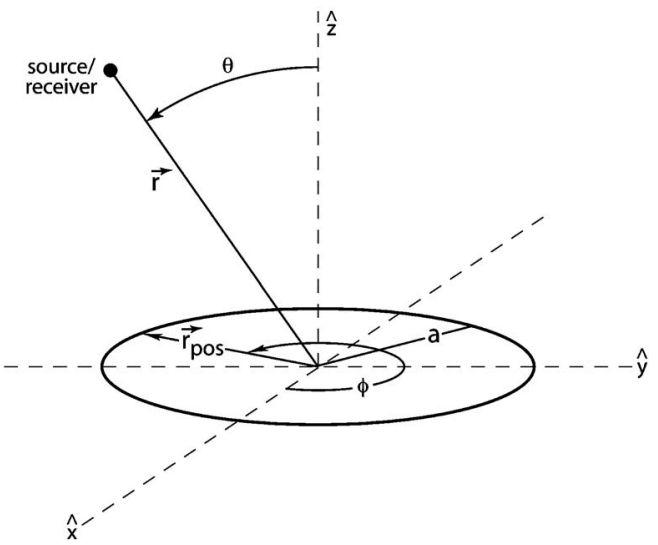

FIG. 2. Diffraction geometry for disk (backscattering). The source/receiver and disk are in the $y z$ and $x y$ planes, respectively.

$$
\begin{aligned}
& \hat{r}_{i}=\sin \theta \hat{j}-\cos \theta \hat{z}, \\
& \hat{r}_{r}=-\sin \theta \hat{j}+\cos \theta \hat{z} \text { (backscatter), }
\end{aligned}
$$

where $a$ is the radius of the disk, $\hat{i}, \hat{j}$, and $\hat{k}$ are the unit vectors associated with the $x, y$, and $z$ axes, respectively, and the source/receiver is in the $y z$ plane.

Inserting these quantities into Eq. (8) gives the expression for the diffracted pressure in the back direction,

$$
P_{\text {diff }}=P_{\text {diff }}^{(\infty, \mathrm{PW})}\left(k / 2 \pi r_{r}^{(T)}\right)^{1 / 2} e^{-i \pi / 4} a I,
$$

where

$$
I \equiv \int e^{i 2 k a \sin \theta \sin \phi} d \phi .
$$

The separate contributions to $I$ from the trailing and leading edges are determined by integrating over the ranges $0 \leqslant \phi$ $\leqslant \pi$ and $\pi \leqslant \phi \leqslant 2 \pi$, respectively:

$$
I=\pi J_{0}(2 k a \sin \theta) \pm i 2 s_{0,0}(2 k a \sin \theta)
$$

where the "+" and "-" signs correspond to the trailing and leading edges, respectively. The terms $J_{0}$ and $s_{0,0}$ are the Bessel and Lommel functions of order zero, respectively.

b. Thin impenetrable disk Evaluation of Eq. (15) requires use of an expression for $P_{\text {diff }}^{(\infty, P W)}$ describing the diffraction by an infinitely long edge. Morse and Ingard (1968) present an exact expression for the total field describing the bistatic diffraction by an impenetrable, infinitely long straight knife-edge due to an incident plane-wave source [Eq. (8.4.6) of that work]. For the case of backscatter, their equation simplifies to

$$
\begin{aligned}
P_{\mathrm{diff}}^{(\infty, \mathrm{PW})}= & A e^{-i k r_{r}^{(0)}}\left\{E\left[\sqrt{2 k r_{r}^{(0)}}\right]-1\right\} \\
& +A e^{i k r_{r}^{(0)} \cos 2 \Psi} E\left[-\sqrt{2 k r_{r}^{(0)}} \sin \Psi\right],
\end{aligned}
$$

where $A$ is the amplitude of the incident plane wave and $\Psi$ is defined in Morse and Ingard as the angle of the direction of the incident plane wave relative to the normal to the halfplane containing the edge. Here, the incident plane wave, $A \exp \left(-i k r_{r}^{(0)}\right)$, was subtracted from the total field to give the scattered field. For an incident wave traveling in a direction 

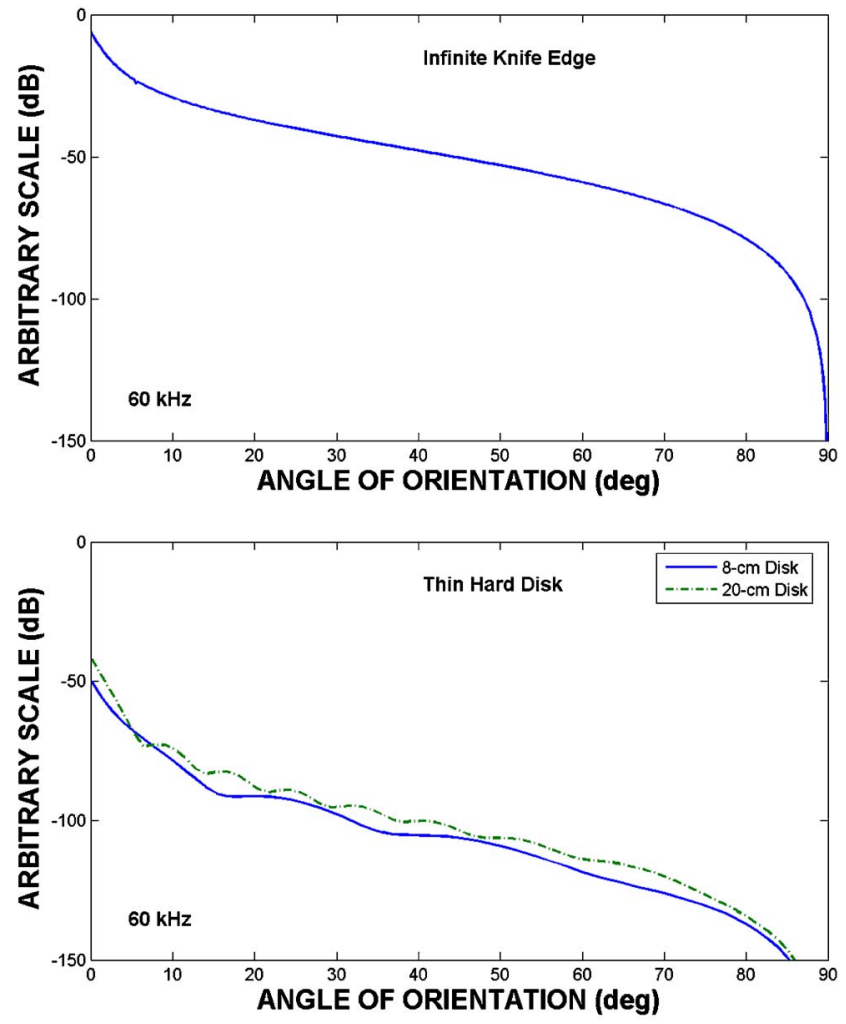

FIG. 3. (Color online) Diffracted echo in backscatter direction from infinitely long, impenetrable straight knife-edge (upper) compared with diffraction in backscatter direction by the leading edge of thin, impenetrable disks (lower). Since the diffracted field spreads differently for the infinitely long edge and the disks, the plots are on arbitrary scales for comparison. The frequency is $60 \mathrm{kHz}$ for all predictions and the diameters of the disks are 8 and $20 \mathrm{~cm}$. The same (exact) expression for $P_{\text {diff }}^{(\infty)}$ [Eq. (18)] was used to produce the upper plot as it was in the lower plots [once integrated through use of Eqs. (15) and (16) to give Eq. (19)], as well as in Fig. 5. The angle $\theta=0^{\circ}$ is normal incidence to flat surface of disk and half-plane associated with the infinite knife-edge, while $\theta=90^{\circ}$ corresponds to edge-on incidence.

normal to the plane, $\Psi=0$, and for edge-on incidence, $\Psi$ $=\pi / 2$. In the disk geometry defined in Fig. 2 of this paper, where $\theta$ is always positive, $\Psi=\theta$ for the leading edge of the disk and $\Psi=-\theta$ for the trailing edge. The function $E[\ldots]$ is defined in Eq. (8.4.3) of Morse and Ingard and can be expressed in terms of Fresnel integrals as shown in Eq. (8.4.5) of that work. Note that Eq. (18) is for all $k r_{r}^{(0)}$ and in the limit of $k r_{r}^{(0)} \gg 1$, the diffracted field varies as $\left(k r_{r}^{(0)}\right)^{-1 / 2}$.

Inserting Eq. (18) into Eq. (15) gives an approximate explicit expression for the diffraction by the edge of a thin, impenetrable disk,

$$
P_{\text {diff }}=A\left(S_{1}+S_{2}\right)\left(k / 2 \pi r_{r}^{(T)}\right)^{1 / 2} e^{-i \pi / 4} a I,
$$

where $S_{1} \equiv e^{-i k r_{r}^{(T)}}\left\{E\left[\sqrt{2 k r_{r}^{(T)}}\right]-1\right\}$

and $S_{2} \equiv e^{i k r_{r}^{(T)} \cos 2 \theta} E\left[ \pm \sqrt{2 k r_{r}^{(T)}} \sin \theta\right]$;

where the "+" and "-" signs in $S_{2}$ correspond to the trailing and leading edges, respectively. Evaluation of Eq. (19) for the leading edge of two disks shows that the diffraction by the edges is a strong function of orientation of the disk and the dimensionless product, $k a$ (Fig. 3). Also, because of the curvature of the disk, the dependence of the diffraction upon $k a$ and orientation angle $\theta$ tends to have an oscillatory component [versus the smoothly varying predictions for the infi- nite knife-edge from Eq. (18)] because of the varying numbers of Fresnel zones occupying the perimeter of the disk.

c. High-frequency limit to circular disk $(k a \gg 1)$. In order to further investigate the diffraction by the edge(s) of a disk, the high-frequency limit is explored. In this case, the frequency is high relative to the radius (i.e., $k a \gg 1$ ). Using the method of stationary phase and dividing the integral in Eq. (16) into sections corresponding to the leading and trailing edges of the disk (one stationary point per section), the integral becomes

Leading edge: $\pi \leqslant \phi \leqslant 2 \pi$

$$
I=\sqrt{\frac{\pi}{k a \sin \theta}} e^{i \pi / 4} e^{-i 2 k a \sin \theta} ;
$$

Trailing edge: $0 \leqslant \phi \leqslant \pi$

$$
I=\sqrt{\frac{\pi}{k a \sin \theta}} e^{-i \pi / 4} e^{i 2 k a \sin \theta} .
$$

In each of these cases, the integral is shown to vary inversely with the square root of the product of $k a$ and $\sin \theta$. Note that the factor of $a^{-1 / 2}$ is offset by the factor of $a$ in the numerator of Eq. (15), resulting in the diffracted field increasing with $a^{1 / 2}$.

\section{First Fresnel zone and effective length}

The first Fresnel zone plays an important role in the diffraction by edges. The radius of the first Fresnel zone of a plane-wave source incident upon an infinitely long straight line and received in the backscatter direction by a point receiver is $\sqrt{r_{r}^{(0)} \lambda}$. Similarly, the radius of the first Fresnel zone of a plane-wave source incident upon a curved finite-length line with a constant radius of curvature $a$ and received in the backscatter direction by a point receiver in the far field of the line and at high frequencies is $\sqrt{a \lambda / 2}$ (where the curvature is in the plane containing the direction of the incident field and the line is bent symmetrically toward or away from the source/receiver). By inserting either of those expressions for the radius of the first Fresnel zone into the term $L$ (length) of the diffracted pressure in the backscatter direction due to a straight finite edge at normal incidence [Eq. (9)], the result will be the diffracted pressure in the backscatter direction for those corresponding cases of an infinitely long straight edge or leading or trailing edge of a disk at edge-on incidence (to within a phase factor). Thus, the effective size of the infinitely long straight edge and edge of a disk is the radius of the first Fresnel zone.

\section{NUMERICAL CALCULATIONS-THIN IMPENETRABLE DISK}

In order to understand the diffraction by a deformed edge under ideal conditions, the T-matrix approach was used to predict the diffraction by thin, impenetrable disks. The T-matrix method is a formally exact numerical solution to the wave equation and is routinely used as a basis for comparison for other approaches. The formulation used is based on the work of Kristensson and Waterman (1982), with details of its implementation given in Norton et al. (1993). 
The T-matrix calculations were computed in the frequency domain and then converted into the time domain through the use of a fast Fourier transform (FFT). The requirement for sampling in the frequency domain was such that the return from the leading and trailing edge in the time domain is separated by at least one time step. Based on the maximum $k a$ used (64), this resulted in the minimum incident angle, capable of resolving the two returns, of approximately six degrees. This maximum $k a$ was kept the same for both disks. Thus, the maximum frequency and delta frequency were different for each disk.

No special windows were applied to the T-matrixgenerated frequency response prior to transforming to the time domain. The rectangular (default) window that results from performing finite Fourier transforms causes the $\operatorname{sinc}(t)$ function to be convolved with the true impulse response. Finally, the numerical results were normalized by the magnitude of the image reflection that occurs in the axial geometry.

The highest order of spherical Hankel and Bessel functions needed for convergence was dependent upon $k a$ [Norton, et al. (1993)]. The higher the $k a$ the more terms required for convergence. For numerical implementation the following rule was used: The highest order used was equal to two times the value of $k a$ (minimum of ten). The maximum azimuthal index used equaled the value of $k a$ (minimum of five). The numbers used at low $k a$ were more than sufficient for convergence, but in order to insure convergence at high $k a$ these relationships were used. No problems were observed throughout the numerical computations and convergence was obtained for all values of $k a$. All computations were carried out on a SGI Altix super computer.

\section{LABORATORY EXPERIMENT-THICK ELASTIC DISK}

The measurements of acoustic diffraction by the disks were part of a larger effort to understand the scattering by benthic shells. In a laboratory experiment conducted in fall 2002 , the scattering by a wide range of metal disks as well as benthic shells was measured. The importance of the diffraction by the outer edge of the shells was identified and comparisons were made between the diffraction by the edge of each shell and the diffraction from the double edge of a disk with outer dimensions comparable to those of the shells (Stanton and Chu, 2004). In this paper, the results from the measurements involving a subset of the disks are reported and analyzed. These disks are constructed of aluminum with a range of diameters $(6-20 \mathrm{~cm})$ at a constant thickness of $1.9 \mathrm{~mm}$. Another set of disks in which the thickness was varied at a constant diameter is analyzed in Chu et al. (2007) where multiple diffraction between the edges is studied. Details of the experimental setup and measurement procedure are given in Stanton and Chu (2004) and in the references cited in that paper, and are briefly summarized below.

The experiments were conducted in a tank of fresh water using two closely spaced transducers, one as transmitter and the other as a receiver. A broadband chirp (linear frequency modulated) signal spanning the frequency range $40-95 \mathrm{kHz}$ was transmitted. The individual targets were $3.0 \mathrm{~m}$ from the transducers and were rotated over the range $0^{\circ}-360^{\circ}$ in $1^{\circ}$ increments. The echoes were processed both in the time and frequency domains. In the time domain, the echoes were temporally compressed through a cross-correlation technique resembling matched-filter processing so that echoes from features of the targets could be resolved. The range resolu-

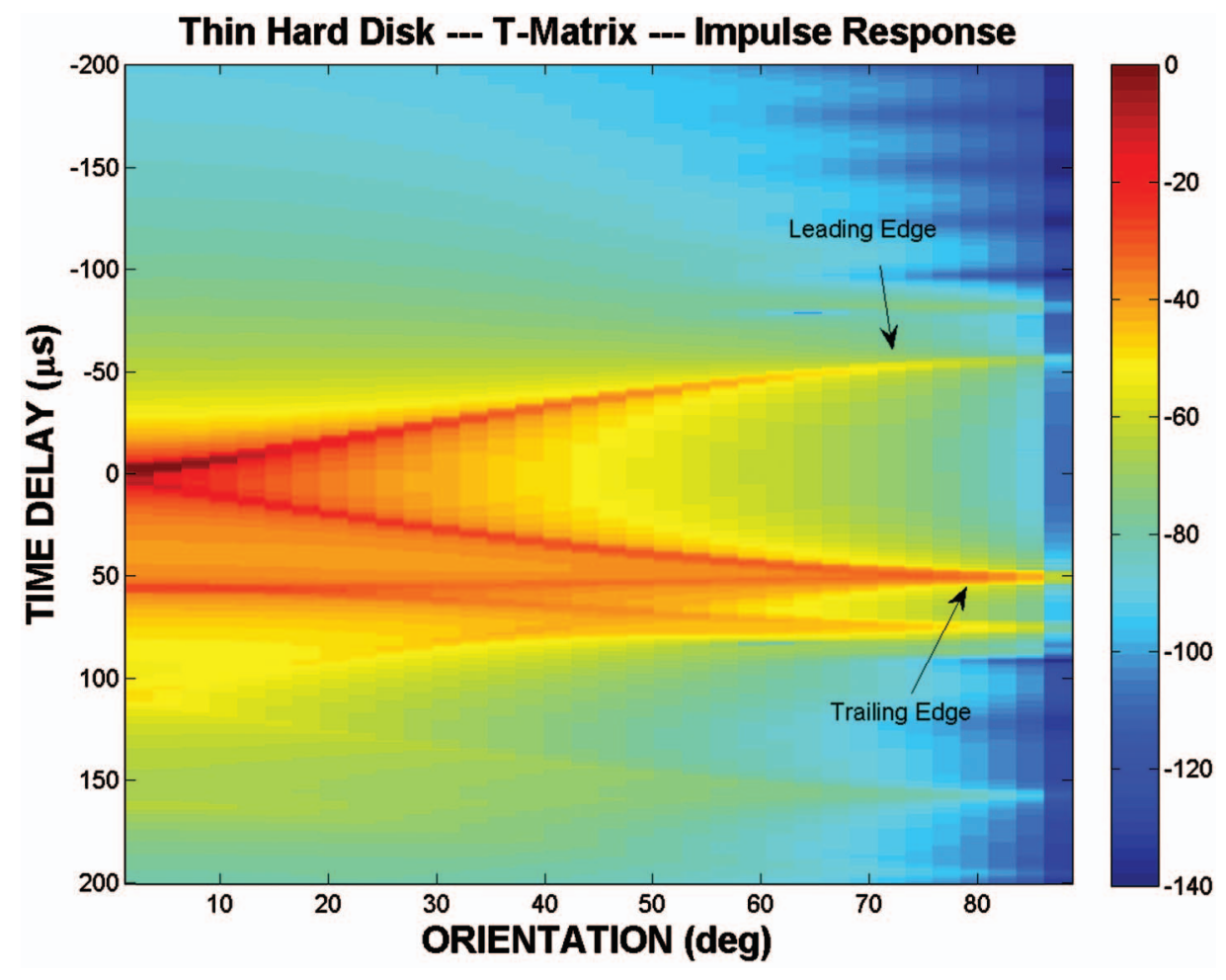

FIG. 4. Impulse response in backscatter direction for 8 -cm-diameter thin disk as calculated with the T-matrix method. Normal incidence is $0^{\circ}$ and edge-on incidence is $90^{\circ}$. The calculations were over the range $2.5-87.5^{\circ}$. The color scale is in decibels relative to the maximum value of the entire plot. The time delay of $0 \mu \mathrm{s}$ corresponds to the center of the disk. 
tion achieved through this signal processing approach is about $2 \mathrm{~cm}$, which corresponds to the inverse bandwidth of the system.

\section{RESULTS}

\section{A. Thin, impenetrable disk}

\section{General observations of numerical predictions}

The impulse response for a 8-cm-diameter thin disk (impenetrable knife-edge) was calculated with the T-matrix method (Fig. 4). The calculations were performed in the backscatter direction and over orientations of the disk spanning the range $2.5-87.5^{\circ}$. (i.e., near normal incidence to near edge-on incidence). The predictions show the diffraction echoes from the edges following the expected sinusoidal pattern throughout the range of orientations. The strength of the backscattered echo from the leading edge is shown to decrease as the orientation angle approaches $90^{\circ}$, which is consistent with the fact that diffraction by a knife edge at edge-on incidence is zero. There is also a strong echo with an arrival time that remains nearly constant at about $55 \mu \mathrm{s}$ throughout the entire range of orientations. This time corresponds to a wave traveling along one face of the disk and across the center (i.e., a travel distance equal to the diameter of the disk).

Partial wave target strengths (PWTS) of the leading edge echoes were calculated with the T-matrix method for the $60-\mathrm{kHz}$ component of the impulse response for two thin, impenetrable disks of different diameters $(8$ and $20 \mathrm{~cm})$. These parameters were chosen for comparison with the laboratory data collected. Here, the PWTS is the target strength that is calculated from echoes from portions of a target, as defined in Chu and Stanton (1998). The leading edge echo is shown to decrease for angles away from normal incidence, and dropping precipitously near edge-on angles (Fig. 5). Also, the echoes are uniformly stronger for the larger disk.

\section{Comparison with deformed knife-edge model}

The deformed edge formulation is evaluated for the thin impenetrable disk, using the exact solution from Morse and Ingard (1968) for the infinite knife-edge [Eq. (19)], and compared with the T-matrix calculations (Fig. 5). The deformed edge predictions for the leading edge of the disk possess the same general trends as with the T-matrix method, as they show a decrease with echo level for angles away from normal incidence, as well as show an increase in level for the larger disk.

The discrepancies between the two sets of predictions are relatively small for angles of orientation greater than about $20^{\circ}$ away from normal incidence. The deformed edge predictions show a more pronounced series of oscillations in the plot of PWTS versus angle than those from the T-matrix predictions.

Also, the calculations from both methods show about a 4-dB difference between the cases of the 8-cm-diameter disk and 20-cm-diameter disk for the higher angles. This is consistent with the limiting form of the deformed edge prediction, where the diffracted field varies as $a^{1 / 2}$ when there are many Fresnel zones occupying the edge (i.e., conditions under which the method of stationary phase can be used) as discussed in Sec. II B 2 c.

\section{B. Thick, elastic disk}

\section{General observations of laboratory data}

The temporally compressed echoes as measured in the laboratory contain resolved echoes from both the leading and trailing double edges of the disks (Fig. 6). Since the range resolution of the system is $2 \mathrm{~cm}$, then the individual edges within each double edge pair are not resolvable. In addition to the edge-diffracted echoes, there are strong echoes from the flat surfaces of the disks at normal incidence to the disk face, as well as echoes associated with circumnavigations around the disk (double edge to flat surface to double-edge path) arriving after the trailing edge echoes. There is overlap between the trailing double-edge echoes and other echoes for much of the range of orientation, preventing quantitative interpretation of the trailing edge echoes. The leading doubleedge diffracted echoes are easily resolved from the other types of echoes over a wide range of angles, and will there-
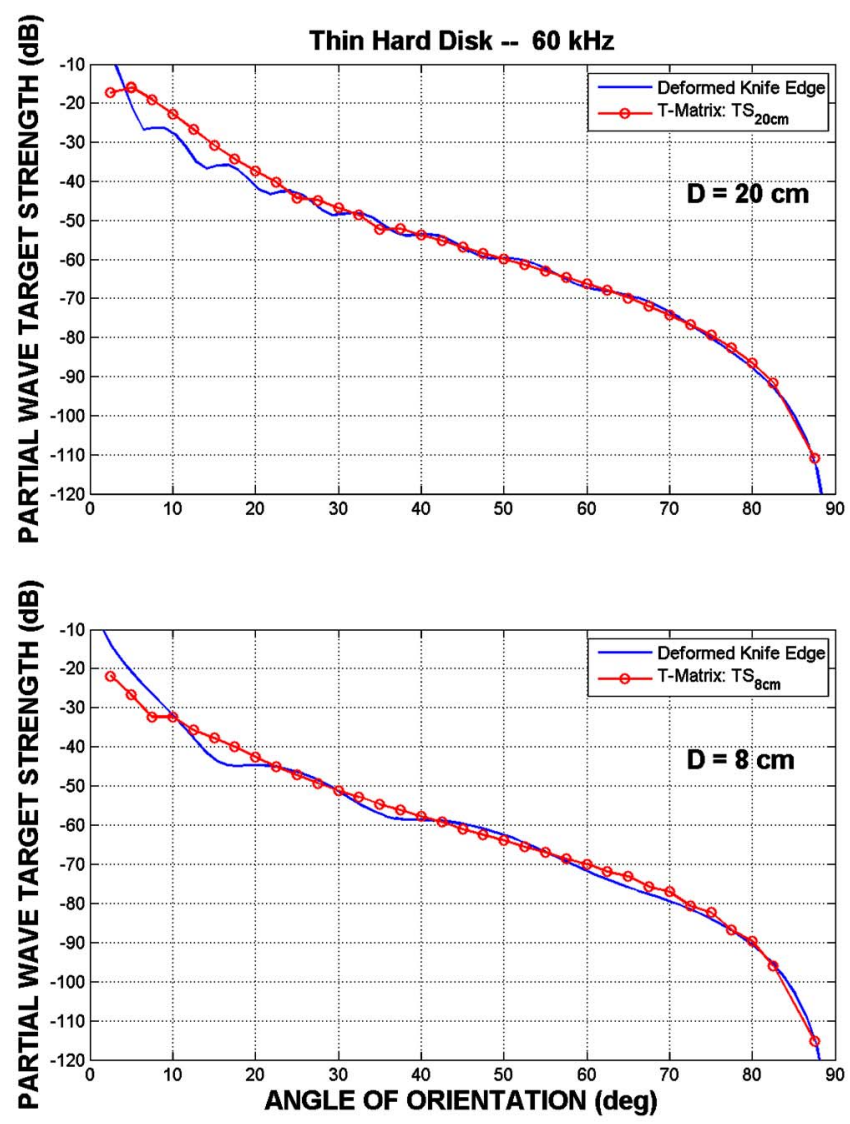

FIG. 5. (Color online) Comparisons between T-matrix and deformed-edge calculations for thin, impenetrable disks of diameters $20 \mathrm{~cm}$ (upper) and $8 \mathrm{~cm}$ (lower). The partial wave target strength (PWTS) of the diffraction by leading edge only is calculated in each case. As in Fig. 3, Eq. (19) was used for the deformed edge calculations, based on an exact solution to the infinite knife-edge. The leading edge echo was numerically separated from the trailing edge echo in the impulse response time series in the T-matrix calculations, although there was difficulty resolving the two echoes (hence resulting in some contamination) for angles below about $20^{\circ}$. All calculations involved $60 \mathrm{kHz}$ for later comparison with the laboratory data. 


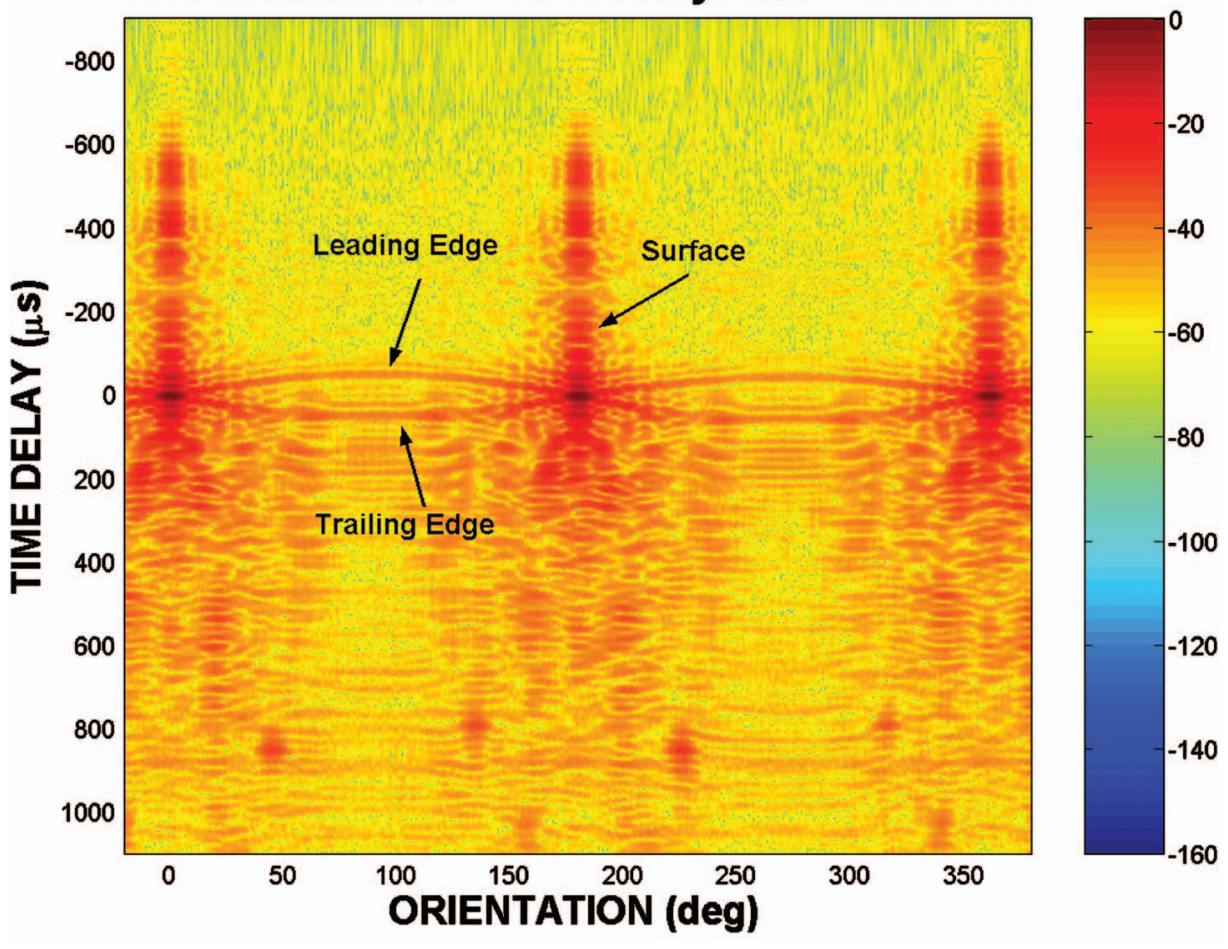

FIG. 6. Temporally compressed echo measured in backscatter direction versus orientation for 8-cm-diameter aluminum disk submerged in water. The disk is $1.9 \mathrm{~mm}$ thick. Normal incidence echoes (at $\theta=0^{\circ}, 180^{\circ}$, and $360^{\circ}$ ), leading and trailing doubleedge echoes, and circumnavigated echoes are resolved. The circumnavigated waves occur at approximately $110 \mu$ s after the trailing edge echoes. Other echoes arrive near the circumnavigated echoes and are out of the scope of this analysis. The color scale is in decibels relative to the maximum value of the entire plot. Apparent echoes at normal incidence arriving at negative time delays are actually processing sidelobes from the large zerotime-delay echoes. The abbreviated terminology "leading edge" and "trailing edge" correspond to the more rigorous description "leading double edge" and "trailing double edge." From Stanton and Chu (2004).

fore be the focus of the analysis. Partial wave target strengths of the leading double-edge echoes were calculated for various spectral components of the signal. The leading doubleedge echo was observed to be generally stronger for orientations closer to normal incidence (Fig. 7). Also, the leading double-edge echo tended to increase with increasing diameter of the disk (Fig. 8).

\section{Comparisons with models}

The measured diffracted echoes from the leading double edge of the elastic disk are generally much larger than those predicted for the thin, impenetrable disk (knife-edge) using either modeling approaches, especially at the larger angles (Figs. 5 and 7). For example, for angles of $40^{\circ}$ away from normal incidence, the observed levels for the 8- and 20-cm-diameter-thick disks are several decibels above the corresponding knife-edge predictions. For angles approaching $90^{\circ}$ away from normal (i.e., edge-on incidence), the observed (thick-disk) levels are tens of decibels higher, as the predicted knife-edge echoes approach $-\infty \mathrm{dB}$. Given the latter discrepancy, the thickness of the disk needs to be taken into account. Of course, the most rigorous treatment would also require accounting for the elastic properties of the disk.

The scattering by the double edge of an elastic disk is complex. In addition to diffraction by the edges (including higher-order diffractions between the edges), there is the potential for conversion of the signal into elastic waves at the boundary. Given that the disks are relatively thin (i.e., $k w$ is comparable to or smaller than unity, where $w$ is the thickness), then the conversion effects on the face of the edge might be small. Also, since the acoustic impedance of aluminum in water is much greater than unity, then there will be little penetration of the incident signal into the disk. There- fore, as a first approximation, the scattering by the double edge will be modeled as the pure diffraction by the two edges.

The data were compared with predictions using a deformed double-edge diffraction formulation by incorporating $P_{\text {diff }}^{(\infty, \text { PS })}$ of Chu et al. (2007) into Eq. (7) of this paper, and evaluating for the disk geometry. In this case involving a point source, the diffracted signal is given by a modified form of Eq. (15) by replacing $P_{\text {diff }}^{(\infty, P W)}$ with $P_{\text {diff }}^{(\infty, P S)}$ and multiplying the right-hand side by $\sqrt{2}$. The Chu et al. formulation involves an impenetrable, infinitely long, straight double edge and is based on the work of Pierce (1974), where the diffraction by a truncated wedge is described. Pierce derived formulas for this case through second-order diffraction. Chu et al. improved the accuracy of the second-order diffraction predictions as well as included all higher orders of diffraction. The expression for the term $I$ in Eq. (16) was used to account for the leading double edge only.

The model predictions were based principally on measured dimensions of the disks, although there were parameters empirically determined from the data from one disk to describe multiple diffraction for all of the disks (Chu et al. 2007). As mentioned earlier, the transmitting and receiving transducers are not collocated, which results in a deviation from the true backscatter direction of an amount of $6.3^{\circ}$. The predictions took into account this deviation, by evaluating $P_{\text {diff }}^{(\infty, \text { PS })}$ for bistatic scattering. The predictions incorporating bistatic scattering differed by less than $1 \mathrm{~dB}$ relative to true backscatter.

There is generally agreement between the predictions and both the trend and much of the structure of the data over most conditions. For example, there is general agreement concerning the trend of decreasing PWTS versus orientation 
Thick Elastic Disk - $60 \mathrm{kHz}$

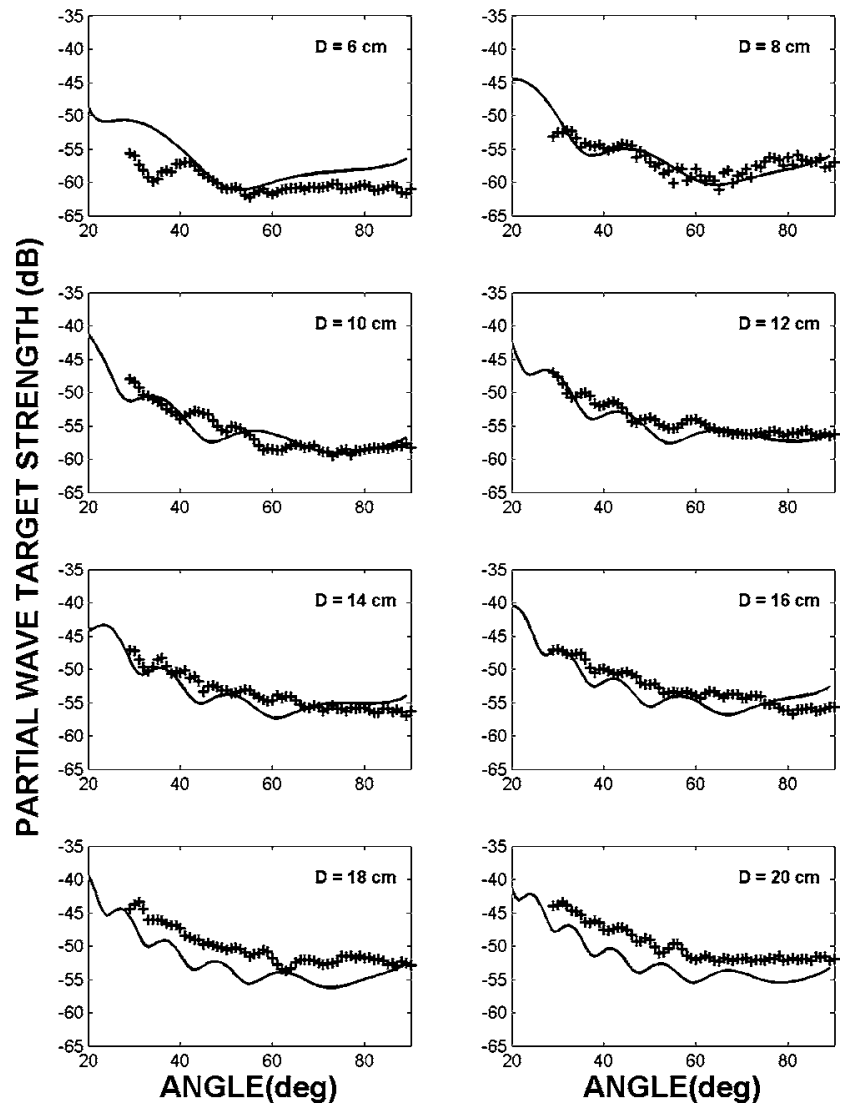

FIG. 7. Partial wave target strength (PWTS) of leading double-edge diffracted $60-\mathrm{kHz}$ echo versus orientation angle for aluminum disks of various diameters. Predictions are given by the solid lines and laboratory data are given by the "+." The diameters of the disks range from 6 to $20 \mathrm{~cm}$, each with a thickness of $1.9 \mathrm{~mm}$. The $0^{\circ}$ angle corresponds to normal incidence to the flat surface of the disks, while $90^{\circ}$ corresponds to edge-on incidence. The angle $\theta$ is illustrated in Fig. 2. The predictions are based on a formulation from Chu et al. (2007) that describes the diffraction by an impenetrable infinitely long, straight double edge. That formula is incorporated into the deformed edge line integral in this paper.

angle of disk (Fig. 7). In that same comparison only some of the observed structure in the data is predicted by the model. For example, all of the structure is predicted for the 8 -cm-diameter disk. However, for the 10-cm-diameter disk, the structure in the range of angles $40^{\circ}-60^{\circ}$ is not predicted. For the disks of higher diameter, both predictions and obser- vations show a more rapid set of oscillations in the pattern of PWTS versus angle, although the respective patterns do not necessarily coincide with each other. There is also very good agreement between predictions and the data concerning the increasing trend of PWTS versus diameter of disk at edge-on incidence (angle of $90^{\circ}$ in Fig. 8). At this angle, both the data and predictions increase by about $5 \mathrm{~dB}$ as the disk diameter is increased from 6 to $20 \mathrm{~cm}$, which is consistent with the deformed edge prediction that the diffracted field varies as $a^{1 / 2}$ (Sec. II B 2 c). The predicted levels tend to depart from observations for angles approaching normal incidence to the disk face and larger diameters (angles of $40^{\circ}$ and $60^{\circ}$ in Fig. 8 ). There was also reasonable agreement between predictions and data in the study of diffraction as a function of thickness of disk (not shown). Those results are presented in Chu et al. (2007) as part of a study of multiple diffraction.

\section{DISCUSSION}

The general agreement between the deformed edge predictions and the numerical calculations and laboratory data over much of the range of conditions indicates that the approximate approach of the line-integral method to estimate effects due to deformations of the edge has merit. The most significant approximation in the predictions involved the approximation of basing the formulation, in part, on a solution involving infinitely long edges. Phase shifts using the lineintegral approach were assigned to the local field or diffraction per unit length to account for deviations of the edges from a straight line. With this approximation, it is anticipated that the approach is generally only valid for slowly varying deformations and for geometries involving near-normal incidence to the edge. However, the disks involve a wide range of angles of the edge relative to the incident acoustic signal, giving rise to conditions of possibly significant error. For each orientation, although there was a wide range of angles (corresponding to different points along the perimeter of the disk), there was always a section of the disk in which the incident angles were near normal to the tangent of the edge-- that is, the section of perimeter closest to the transceiver. This section contains the first Fresnel zone of the acoustic signal which, at these high frequencies, will dominate the echo. Thus, although the predictions for the portions of the edge outside of the first Fresnel zone may contain
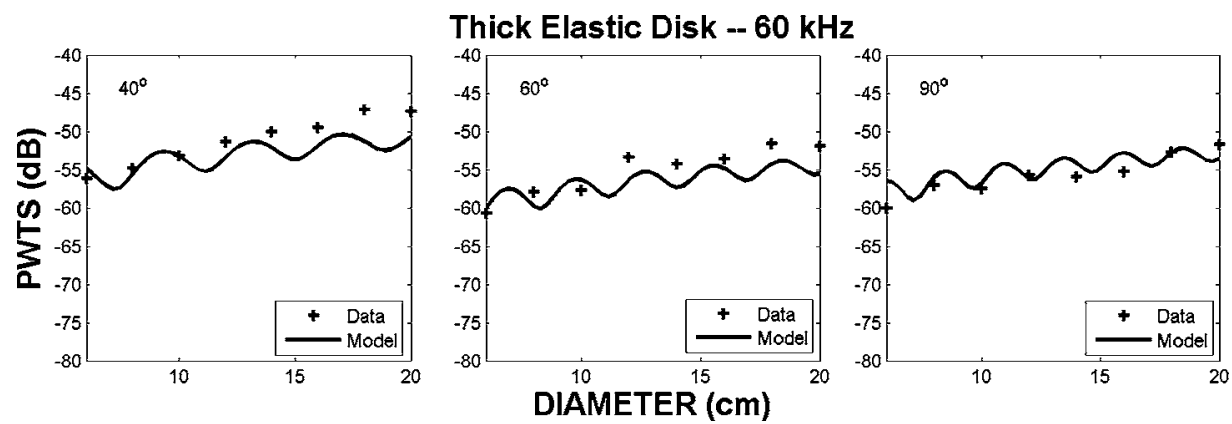

FIG. 8. PWTS of leading double-edge echo versus diameter of aluminum disk at $60 \mathrm{kHz}$ and for three orientation angles. Model predictions are given by the solid lines and laboratory data are given by the "+." The thickness of the disks is $1.9 \mathrm{~mm}$. The angle $\theta$ is illustrated in Fig. 2. The predictions use the same model (impenetrable deformed double edge) as in Fig. 7. The data for the $90^{\circ}$ angle follow the trend of varying by $a^{1 / 2}$, as predicted by the deformed edge model, over the entire range of diameters. 
significant error, the errors tend to be canceled out through the alternating signs of the adjacent Fresnel zones. Given the occurrence of this phenomenon, the potentially significant errors in the approach are not realized with this particular scattering geometry.

Certainly, the purest test of the line-integral formula involved the thin, impenetrable disk. Here, an exact formula was used for the infinite knife-edge and the comparison was made with the T-matrix calculations which are exact. Thus, the principal source of error was in the fundamental approximation of the line-integral formula where the local diffraction per unit length was based on the infinite edge formulation. The comparisons showed that the line-integral approach was to within about $2 \mathrm{~dB}$ of the T-matrix calculations for the angles of incidence greater than $20^{\circ}$ from normal incidence. There were greater discrepancies for smaller angles. These latter differences can be attributed, at least in part, to the fact that it was increasingly more difficult at the small angles to numerically resolve the echoes from the leading and trailing edges in the T-matrix calculations.

A much less pure, but interesting, test involved the comparison of the formulation with the laboratory data involving thick, elastic disks. Here, in addition to the fundamental limitation of the formulation discussed above, there were sources of error involving the differences between diffraction by an elastic body and an impenetrable one, and the fact that the infinite double-edge formula used in the integral was approximate. With these additional sources of error, the discrepancies were generally up to about $5 \mathrm{~dB}$. Quantification of the error associated with elastic effects are out of the scope of this present study, although the error associated with the double-edge formula is discussed in Chu et al. (2007).

\section{CONCLUSIONS}

A general approximate formula was derived to predict diffraction from deformed edges of finite length using a lineintegral approach. The formula is general, as it is written for arbitrary deformations and is based on an arbitrary infinite edge formula. There was reasonable agreement between predictions using this formula and exact numerical predictions for a thin, impenetrable disk and laboratory data involving thick, elastic disks. For the conditions under which the sources of error in the analysis were at a minimum, i.e., angles greater than about $20^{\circ}$ from normal incidence of the thin, impenetrable disks, predictions using the formulation were to within about $2 \mathrm{~dB}$ of the exact solution. Deviations were expectedly greater (about $5 \mathrm{~dB}$ ) with the thick, elastic disk as the infinite double-edge formula was approximate and elastic effects were not taken into account. Although there is potential for significant error for any finite-length edge geometry due to the fact that the formula is based, in part, on one associated with infinitely long edges, significant error was not realized for the scattering geometries in this paper due to the dominance of the first Fresnel zone and cancellation of the errors associated with higher-order
Fresnel zones. Given the wide range of conditions under which this line-integral approach appears to be valid, there is great utility in the approach.

\section{ACKNOWLEDGMENTS}

The authors are grateful to the anonymous reviewers for their thoughtful advice that led to significant improvements of this paper. The authors are also grateful to Shirley Barkley, Jayne Doucette, and Craig Johnson, all from the Woods Hole Oceanographic Institution (WHOI), Woods Hole, MA, for preparation of the manuscript to this paper, drawing certain figures, and for construction of the disks, respectively. This research was supported by the U.S. Office of Naval Research (Grant No. N00014-02-0095), WHOI, and by a grant of computer time at the U.S. Department of Defense High Performance Computing Shared Resource Center (Naval Research Laboratory, Washington, DC).

Bowman, J. J., Senior, T. B. A., and Uslenghi, P. L. E. (1987). Electromagnetic and Acoustic Scattering by Simple Shapes (Hemisphere, New York).

Chu, D., and Stanton, T. K. (1998). "Application of pulse compression techniques to broadband acoustic scattering by live individual zooplankton," J. Acoust. Soc. Am. 104, 39-55.

Chu, D., Stanton, T. K., and Pierce, A. D. (2007). "Higher-order acoustic diffraction by edges of finite thickness," J. Acoust. Soc. Am. 122, 3177.

Hefner, B. T. (2000). "Acoustic backscattering enhancements for circular elastic plates and acrylic targets, the application of acoustic holography to the study of scattering from planar elastic objects, and other research on the radiation of sound," Ph.D. thesis, Washington State University.

Hefner, B. T., and Marston, P. L. (2001). "Backscattering enhancements associated with the excitation of symmetric Lamb waves on a circular plate: Direct and holographic observations," ARLO 2, 55-60.

Hefner, B. T., and Marston, P. L. (2002). "Backscattering enhancements associated with antisymmetric Lamb waves confined to the edge of a circular plate: Direct and holographic observations," ARLO 3, 101-106.

Keiffer, R. S., Novarini, J. C., and Norton, G. V. (1994). "The impulse response of an aperture: Numerical calculations within the framework of the wedge assemblage method," J. Acoust. Soc. Am. 95, 3-12.

Kristensson, G., and Waterman, P. C. (1982). "The $T$ matrix for acoustic and electromagnetic scattering by circular disks,” J. Acoust. Soc. Am. 72, 1612-1625.

Medwin, H. (1981). "Shadowing by finite noise barriers," J. Acoust. Soc. Am. 69, 1060-1064.

Medwin, H., Childs, E., Jordon, E. A., and Spaulding, R.A. Jr. (1984). "Sound scatter and shadowing at a seamount: Hybrid physical solutions in two and three dimensions," J. Acoust. Soc. Am. 75, 1478-1490.

Menounou, P., and You, J. H. (2004). "Experimental study of the diffracted sound field around jagged edge noise barriers," J. Acoust. Soc. Am. 116, 2843-2854.

Menounou, P., Busch-Vishniac, I. J., and Blackstock, D. T. (2000). "Directive line source model: A new model for sound diffraction by half planes and wedges," J. Acoust. Soc. Am. 107, 2973-2986.

Morse, P. M., and Ingard, K. U. (1968). Theoretical Acoustics (Princeton University Press, Princeton, NJ).

Norton, G. V., Novarini, J. C., and Keiffer, R. S. (1993). "An evaluation of the Kirchhoff approximation in predicting the axial impulse response of hard and soft disks," J. Acoust. Soc. Am. 93, 3049-3056.

Pierce, A. D. (1974). "Diffraction of sound around corners and over wide barriers," J. Acoust. Soc. Am. 55, 941-955.

Skudrzyk, E. (1971). The Foundations of Acoustics (Springer, New York). Stanton, T. K. (1989). "Sound scattering by cylinders of finite length III. Deformed cylinders," J. Acoust. Soc. Am. 86, 691-705.

Stanton, T. K., and Chu, D. (2004). "On the acoustic diffraction by the edges of benthic shells," J. Acoust. Soc. Am. 116, 239-244.

Svensson, U. P., Fred, R. I., and Vanderkooy, J. (1999). "An analytic secondary source model of edge diffraction impulse responses," J. Acoust. Soc. Am. 106, 2331-2344. 